\title{
Radiation processes in the Seyfert galaxy NGC 4151
}

\author{
M. Eriksson \\ Institute of technology, University college of Blekinge, 37179 Karlskrona, Sweden \\ e-mail: meo@bth.se
}

Received 17 August 2012 / Accepted 1 October 2012

\begin{abstract}
Aims. The main aim of this work was to investigate if the radiative process photoexcitation by accidental resonance (PAR) is producing Fe II lines in the UV region of NGC 4151. At the same time I searched for collisionally excited Fe II emission. While doing so, the broad line region (BLR), intermediate line region (ILR) and narrow line region (NLR) contributions to the Mg II resonance doublet were also investigated.

Methods. The focus was on the 2000 to $3000 \AA$ wavelength region, in which decay from both the collisionally and PAR excited Fe II levels would form emission lines. I examined three archived Space Telescope Imaging Spectrograph (STIS) spectra of NGC 4151. Theoretical spectra based on calculated ranges for possible fluxes of the collisionally excited lines and pumping rates through the Fe II channels were then fitted to the observed spectra

Results. I show that the UV region is influenced by PAR and that the Fe II fluorescence lines are clearly needed to explain the spectrum between 2000 to $3000 \AA$. The best fit of the theoretical spectra to the STIS spectra was obtained by assuming a similar radiation power of $\mathrm{Fe}^{+}$ions and $\mathrm{Mg}^{+}$ions.

Conclusions. The PAR is active in the BLR of NGC 4151 and the total power of the Fe II fluorescence is a significant fraction of the total emission in the 2000 to 3000 wavelength region. Therefore it is important to incorporate this in models of NGC 4151 and possibly also in models of other active galactic nuclei. There are also many collisionally excited Fe II lines that are blended by each other and are therefore not individually observable in the NGC 4151 spectra.
\end{abstract}

Key words. atomic processes - line: formation - galaxies: active - galaxies: Seyfert - galaxies: individual: NGC 4151

\section{Introduction}

In active galactic nuclei (AGN) a major part of the emission is nonstellar. The power of these objects is believed to be due to accretion of matter onto a supermassive black hole. In the case of NGC 4151 the mass of the assumed black hole is $(4.6 \pm$ $0.6) \times 10^{7} M_{\odot}($ Bentz et al. 2006). NGC 4151 belongs to an AGN subcategory called Seyfert 1 galaxies, which are characterized by very broad (full width at half maximum, FWHM, of several thousand $\mathrm{km} \mathrm{s}^{-1}$ ) emission lines of permitted lines and narrower emission lines (FWHM of a few hundred $\mathrm{km} \mathrm{s}^{-1}$ ) The corresponding emission regions are then referred to as broad line region (BLR) and narrow line region (NLR). The BLR of Seyfert galaxies normally extends to a few percent of a light year from the core (Peterson et al. 2004) and is associated with high densities. The NLR extends to several parsecs and is associated with much lower densities. From observations of the brightest Seyfert galaxies absorption components in the blue wing have been observed in some of the UV BLR lines. Those are referred to as the UV absorbers. Even though the UV absorbers correspond to transitions also active in the BLR, they are not believed to have the same origin. The radial velocities of the UV absorbers are about $\sim 1500 \mathrm{~km} \mathrm{~s}^{-1}$ (Crenshaw \& Kraemer 2005) while the broad lines have widths about a magnitude larger. Also, the UV absorption seems to originate at distances from about a half to a few light years from the nucleus (Peterson et al. 2004) while the BLR only extends a few percent of a light year (Kraemer et al. 2005). In NGC 4151 a third emission line category has been identified (Crenshaw \& Kraemer 2007), consisting of lines with a FWHM of about $1200 \mathrm{~km} \mathrm{~s}^{-1}$. Those lines stem mainly from the same transitions as the BLR lines and thus make components in the corresponding line structures. Since those components have widths broader than the NLR lines but less wide than the BLR lines, their origin is referred to as the intermediate line region (ILR). Crenshaw and Kraemer furhtermore suggested that the ILR could be the same region as that of the UV absorbers, or at least that those to regions overlap. It is also possible that there is no clear separation between the ILR and BLR. The BLR arises far down in the disk wind (Chiang \& Murray 1996). One possibility is that the width of the emission lines depends on where in a deaccelerated wind they are formed. In modeling the spectrum in this work, the ILR components are allowed widths from the $1200 \mathrm{~km} \mathrm{~s}^{-1}$ ILR components observed in permitted UV lines by Kraemer et al (2006) up to the widths of the BLR lines. The various emission regions as well as the considerable variation in ionization degree of the observed emission lines enable in turn strong variations in the radiation processes. Another type of astronomical objects that also has several different emission regions of different natures and wide ranges of ionization degrees are symbiotic stars. Even if symbiotic stars are smaller by many magnitudes than the AGN, radiation processes observed in the symbiotic stars could also occur in AGNs. One process with a very strong impact on the UV spectrum of symbiotic stars is photoexcitation by accidental resonance (PAR) (Eriksson et al. 2006). The most striking effect of the PAR process in symbiotic stars is the large number of strong Fe II fluorescence lines in the 2300-2800 $\AA$ region. In the same wavelengt there is an emission "bump" observed in some AGNs (Baldwin et al. 2004) that has not been explained yet. In this work I model the pumping of Fe II by the 
Table 1. Absorption lines in the 2000 to $3000 \AA$ A region.

\begin{tabular}{lcc}
\hline \hline Wavelenth & Spectrum & Upper level \\
\hline 2249.88 & Fe II & $\left({ }^{5} \mathrm{D}\right) 4 \mathrm{p}^{4} \mathrm{D}_{7 / 2}$ \\
2260.78 & Fe II & $\left({ }^{5} \mathrm{D}\right) 4 \mathrm{p}^{4} \mathrm{~F}_{9 / 2}$ \\
2344.21 & Fe II & $\left({ }^{5} \mathrm{D}\right) 4 \mathrm{p}^{6} \mathrm{P}_{7 / 2}$ \\
2374.46 & Fe II & $\left({ }^{5} \mathrm{D}\right) 4 \mathrm{p}^{6} \mathrm{P}_{9 / 2}$ \\
2382.77 & Fe II & $\left({ }^{5} \mathrm{D}\right) 4 \mathrm{p}{ }^{6} \mathrm{P}_{11 / 2}$ \\
2586.65 & Fe II & $\left({ }^{5} \mathrm{D}\right) 4 \mathrm{p}^{6} \mathrm{D}_{7 / 2}$ \\
2600.17 & Fe II & $\left({ }^{5} \mathrm{D}\right) 4 \mathrm{p}{ }^{6} \mathrm{D}_{9 / 2}$ \\
2796.25 & $\mathrm{Mg}$ II & $3 \mathrm{p}{ }^{2} \mathrm{P}_{3 / 2}$ \\
2803.53 & $\mathrm{Mg}$ II & $3 \mathrm{p}^{2} \mathrm{P}_{1 / 2}$ \\
2852.96 & $\mathrm{Mg}$ I & $3 \mathrm{~s} 3 \mathrm{p}^{1} \mathrm{P}_{1}$ \\
\hline
\end{tabular}

two strongest UV lines in the spectrum of NGC 4151, H Ly $\alpha$ and the C IV $\lambda 1550$ doublet in the BLR to see whether this could explain the observed emission. The strongest feature in the 2000 to $3000 \AA$ region is the $\mathrm{Mg}$ II $\lambda 2800$ doublet. Since both ionization and excitation energy for the Mg II $3 p$ levels are very similar to those of the Fe II $\left({ }^{5} \mathrm{D}\right) 4 \mathrm{p}$ levels, which are the lowest odd Fe II levels, it is possible that some Fe II radiation also leaves NGC 4151. Thus, the Fe II emission is included in my model spectra of NGC 4151 .

\section{Data}

There are three HST/STIS spectra covering the 2000 to $3000 \AA$ region of NGC 4151 at the MAST archive. Two of these are from a program by Hutchins et al. (1998), who observed NGC 4151 at two different position angles, $221^{\circ}$ on 8 Jan. 1998 and $70^{\circ}$ on 7 May 1998. These authors used an exposure time of $2160 \mathrm{~s}$ at both times. The third spectrum was observed by Mundell et al. (2000) on 24 May 2000 at a position angle of $83^{\circ}$. They used an exposure time of $2825 \mathrm{~s}$. All three spectra were recorded with a $\mathrm{CCD}$ using the G230LB grating with a dispersion of $1.35 \AA$ pixel and a spectral resolution of $2.7 \AA$ (Woodgate et al. 1998). Crenshaw \& Kraemer (2001) estimated the color excess, $E(B-V)$, of NGC 4151 to be 0.02 . I have adopted this value and dereddened the spectra using the reddening curves by Savage \& Mathis (1979). The Space Telescope Imaging Spectrograph (STIS) long slit only covers parts of the nucleus of NGC 4151, which is why the observed differences in the continuum level in the different spectra of NGC 4151 are expected due to the different position angles. To set the continuum level for the three STIS spectra I chose points for which there are no signs of spectral lines. From atomic data together with the appearance of the NGC 4151 spectra four such points were selected, corresponding to $1600,2000,2180$, and $3050 \AA$. The continuum level was then approximated to the best parabolic arc fit to those points. In the 2000 to $3000 \AA$ wavelength region of the STIS spectra of NGC 4151 there are ten absorption lines with instrumental profile, meaning that their widths in the spectra equal the spectral resolution and thus, they are too narrow to measure their widths. All these ten lines are transitions from the ground level of the corresponding ion. Table 1 lists the identifications of these lines. There is no redshift for these lines, which together with their narrow nature indicates an origin within our milky way rather than in NGC 4151. Probably some cold material along the line of sight to NGC 4151 within our own galaxy is responsible for these lines. To avoid misinterpreting the data I measured the column densities of these ions from their absorption lines and simulated this absorption in our model spectra.
Table 2. Emission other than Fe II in the 2000 to $3000 \AA$ A wavelength region.

\begin{tabular}{lccc}
\hline \hline Wavelength & Spectrum & Multiplet & Transition \\
\hline 2320.95 & [O III] & $2 \mathrm{p}^{2}{ }^{3} \mathrm{P}-2 \mathrm{p}^{2}{ }^{1} \mathrm{~S}$ & $1-0$ \\
2323.50 & $\mathrm{C}$ II & $2 \mathrm{~s}^{2} 2 \mathrm{p}^{2} \mathrm{P}-2 \mathrm{~s} 2 \mathrm{p}^{2}{ }^{4} \mathrm{P}$ & $1 / 2-3 / 2$ \\
2324.69 & & & $1 / 2-1 / 2$ \\
2325.40 & & & $3 / 2-5 / 2$ \\
2326.93 & & & $3 / 2-3 / 2$ \\
2328.12 & & & $3 / 2-1 / 2$ \\
2421.77 & [Ne IV] & $2 \mathrm{p}^{3}{ }^{4} \mathrm{~S}-2 \mathrm{p}^{3}{ }^{2} \mathrm{D}$ & $3 / 2-3 / 2$ \\
2427.41 & & & $3 / 2-5 / 2$ \\
2470.22 & [O II] & $2 \mathrm{p}^{3}{ }^{4} \mathrm{~S}-2 \mathrm{p}^{3}{ }^{2} \mathrm{P}$ & $3 / 2-1 / 2$ \\
2470.34 & & & $3 / 2-3 / 2$ \\
2796.35 & $\mathrm{Mg}$ II & $3 \mathrm{~s}^{2} \mathrm{~S}-3 \mathrm{p}{ }^{2} \mathrm{P}$ & $1 / 2-3 / 2$ \\
2803.53 & & & $1 / 2-1 / 2$ \\
\hline
\end{tabular}

\section{The model spectra}

\subsection{Emission lines other than Fe II}

Four lines have been identified in the 2000 to $3000 \AA$ region of NGC 4151 spectra (Nelson et al. 2000). One of the lines is the previously well-studied Mg II resonance doublet $\lambda \lambda 2796$, $2804 \AA$ (Leech et al. 1987). Two of the lines are unblended parity forbidden term transitions within ground configuration of the corresponding ions. Those two lines are from [O II] and [Ne IV] at 2470 and $2427 \AA$. The fourth line is a blend between a parity forbidden line from [O III] at $2320.95 \AA$ and an intercombination multiplet from C II ranging from 2323 to $2328 \AA$. Table 2 lists the transitions that are forming emission lines in the 2000 to $3000 \AA$ region in NGC 4151. The finestructure of the multiplets in Table 2 is unresolved due to the widths of the lines. The UV spectrum of NGC 4151 has been studied in spatially resolved STIS spectra of NGC 4151 in two different slit orientations by Nelson et al. (2000) and then in a third orientation by Crenshaw \& Kraemer (2005). The variation of intensity of the unblended [Ne IV] and [O II] lines is the same with respect to angular distance from the center of NGC 4151 core. Both lines also have the same relative intensity in the two slit orientations. At optical wavelengths there are additional lines, identified as parity forbidden transitions from ions of ionization degrees ranging from zero to six. Those lines are from different elements such as nitrogen, sulphur, oxygen, and neon. The relative intensity of the forbidden lines from the second to the fourth spectra varies in similar fashion both with respect to distance from galactic center and choice of slit orientation. The narrow lines in NGC 4151 originate from a number of clouds (Kaiser et al. 2000). The clouds to the NE of the nucleus have recessional velocities and the clouds to the SW have approaching velocities relative to the nucleus. The majority of the clouds have velocities in the range $|v|<800 \mathrm{~km} \mathrm{~s}^{-1}$. The broadening of the forbidden lines due to the velocity dispersion between the NLR clouds is the same for the lines from different ions. Since this broadening effect is also the most important because of the wide range in cloud velocity, the widths of the forbidden emission lines in our model spectra are the same within $10 \%$, regardless what ions they originate from. A small variance is allowed because the thermal broadening cannot be proven to be completely negligible. The Mg II doublet in NGC 4151 has been well studied already in the International Ultraviolet Explorer (IUE) spectra (Leech et al. 1987). Its profile is known to be affected by different regions in NGC 4151. I have incorporated an ILR component (FWHM $1500 \mathrm{~km} \mathrm{~s}^{-1}$ ) and a broad component 
(FWHM $10000 \mathrm{~km} \mathrm{~s}^{-1}$ ). The physical nature corresponding to the $\mathrm{C}$ II intercombination lines differs from the parity forbidden lines of oxygen and neon. It is therefore difficult to know the exact origin of the C II emission. An additional complication is that the C II multiplet is blended with the forbidden [O III] spectra in the STIS spectra. Because of the questionable nature of the C II emission the intensity and width of the C II lines in our model, spectra have wide allowed ranges. The width of the C II lines are allowed to be between the width of the narrower $\mathrm{Mg}$ II components and the width of the parity forbidden lines from oxygen and neon. Since emission from NLR are observed on both sides of the nucleus, I assumed the systematic redshift of all NLR lines in the 2000 to $3000 \AA$ wavelength region to be the same as the redshift of the galaxy, which is $995 \mathrm{~km} \mathrm{~s}^{-1}$ (Crenshaw et al. 2000).

\subsection{The emission from Fe II $\left({ }^{5} D\right) 4 p$}

For each $3 d^{6} \mathrm{nl}$ configuration the lowest energy levels correspond to the parent term $\left({ }^{5} \mathrm{D}\right) \mathrm{nl}$. This is due to the ground term of Fe III, which is $3 d^{6}{ }^{5} \mathrm{D}$. In collisionally excited plasmas most of the Fe II emission stems from allowed transitions from the lowest odd terms to low-lying even terms. There are six terms of Fe II corresponding to the $3 d^{6}\left({ }^{5} \mathrm{D}\right) 4 \mathrm{p}$ parent term, which involves the lowest energy levels in Fe II from which allowed and semi-forbidden (intercombination) transitions can originate. Lines from those levels are therefore frequently observed and are normally expected to be the first to be observed in emission plasma with $\mathrm{Fe}^{+}$ions. An exception are plasmas with very low densities, $n_{\mathrm{e}} \ll 10^{-5} \mathrm{~cm}^{3}$, for which the $3 \mathrm{~d}^{6} 4 \mathrm{~s}$ levels corresponding to parent terms of higher energy than Fe III ${ }^{5} \mathrm{D}$ have time to decay. The time between collisions needs to be on the order of seconds for any substantial production of parity forbidden lines. Forbidden lines from such transitions are present in planetary nebulae for example. In AGNs the Fe II emission are believed to originate from the broad line regions (Gregory et al. 2010) with higher densities. The 25 different Fe II $3 d^{6}\left({ }^{5} \mathrm{D}\right) 4 \mathrm{p} \mathrm{lev-}$ els have energies ranging from $4.76 \mathrm{eV}$ to $5.91 \mathrm{eV}$, which is not much more than the 4.42 and $4.43 \mathrm{eV}$ for the two $\mathrm{Mg}$ II 3p levels responsible for the Mg II $\lambda \lambda 2796.35,2803.53 \AA$ doublet. The ionization energy of Fe I and $\mathrm{Mg}$ I are also similar, 7.90 and $7.64 \mathrm{eV}$. Figure 1 shows that the fraction of the iron and magnesium atoms are single ionized and excited to a level where allowed UV emission can be formed as a function of temperature. Those fractions are calculated by Boltzmann and Saha equations as follows

$$
\begin{aligned}
& F=\frac{f_{01}}{1+\sum_{i}\left(\prod_{k=1}^{i} f_{k-1, k}\right)} \sum_{i} \frac{g_{i} \mathrm{e}^{-\frac{E_{i}}{k T}}}{Q_{2}(T)} \\
& f_{k-1, k}=\frac{Q_{k}(T)}{Q_{k-1}(T)} \frac{\mathrm{e}^{-\frac{I_{k-1}}{k T}}}{L_{\mathrm{e}}^{3} n_{\mathrm{e}}},
\end{aligned}
$$

where $Q_{i}(T)$ and $I_{i}$ are the partition function and ionization potential of the $i$ th ionization stage. $L_{\mathrm{e}}$ is the de-Broglie wavelength of the electrons in the plasma. Since the abundance of iron is mostly found to be very close to that of magnesium in galaxies (Smith et al. 2009), the observed broad line emission component of the Mg II $\lambda 2800 \AA$ doublet indicates that the UV spectrum of NGC 4151 could be affected by Fe II emission from the $3 d^{6}\left({ }^{5} \mathrm{D}\right) 4 \mathrm{p}$ levels. Decay from the Fe II $\left({ }^{5} \mathrm{D}\right) 4 \mathrm{p}$ terms to the four lowest Fe II terms, $\left({ }^{5} \mathrm{D}\right) 4 \mathrm{~s}{ }^{6} \mathrm{D},\left({ }^{5} \mathrm{D}\right) 4 \mathrm{~s}{ }^{4} \mathrm{D}, 3 \mathrm{~d}^{7} \mathrm{a}^{4} \mathrm{~F}$ and $3 d^{7} a^{4} P$, results in many lines in the 2000 to $3000 \AA$ region, Fig. 2. In Fig. 3 the theoretical spectrum of the collisionally excited Fe II lines, assuming FWHM of $15000 \mathrm{~km} \mathrm{~s}^{-1}$ as observed

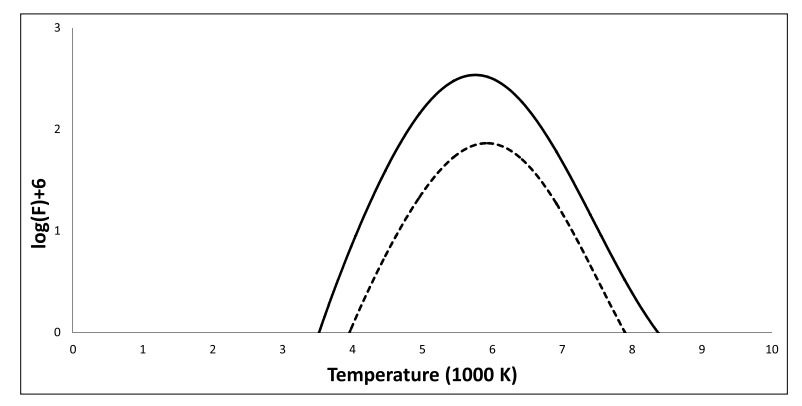

Fig. 1. Fraction of magnesium and iron in all ionizing stages that are in the $\mathrm{Mg}$ II $3 p$ or Fe II $\left({ }^{5} \mathrm{D}\right) 4 \mathrm{p}$ states. The solid line is for magnesium and the dotted is for iron. The partition functions for the magnesium ionization stages are approximated with statistical weights for the corresponding ground levels. This is a good approximation at temperatures below $10^{4} \mathrm{~K}$ because there is now fine structure in the ground term of the three first ionization stages. For iron there are many levels of low excitation energy and the partition functions used here are from Halenka $\&$ Madej (2002). Below $10^{4} \mathrm{~K}$ the partition functions are almost constant through different lowering of the ionization energies. In this graph a lowering of the ionization energy of $0.05 \mathrm{eV}$ and an electron density of $10^{9} \mathrm{~cm}^{-3}$ is adopted.

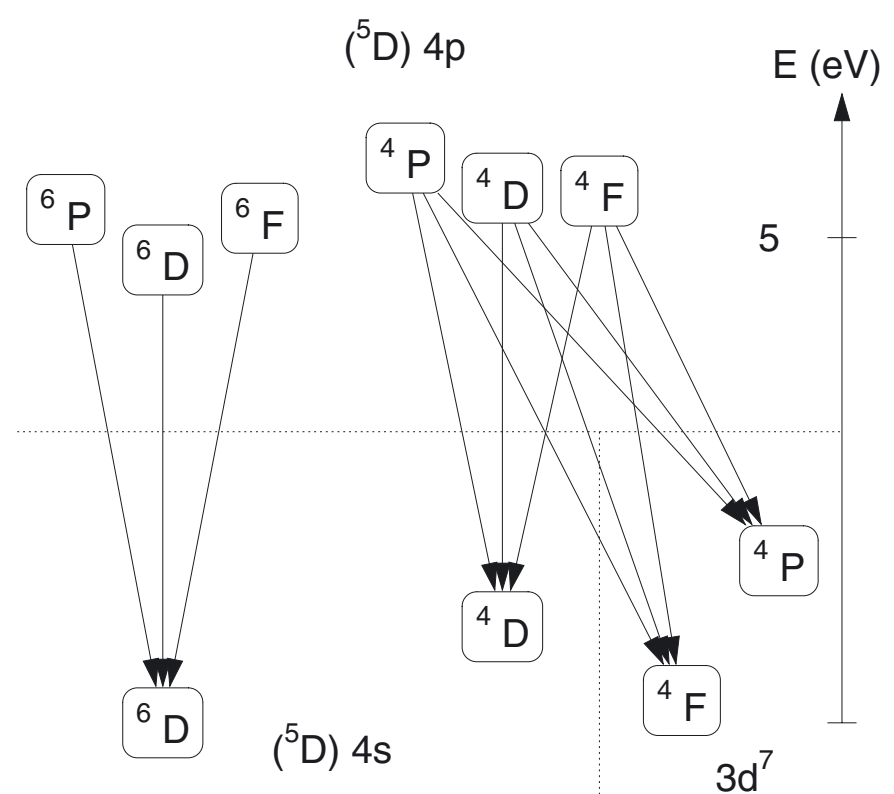

Fig. 2. Terms responsible for the collision excited Fe II emission in the 2000-3000 $\AA$ wavelength region. The 12 resulting allowed multiplets are indicated with arrows. There are also 12 additional intercombination multiplets that are incorporated in my model spectra.

in the BLR of NGC 4151, is presented. From the previous discussion in this section one can expect the total integrated intensity from collisionally excited Fe II at UV wavelengths to be of the same magnitude as the broad component of the Mg II resonance doublet. I therefore restricted the total intensity of the Fe II collisionally excited lines in my model spectra to differ by less than a factor of two from the intensity of the broad component of the Mg II resonance doublet. The width of the Fe II lines is restricted to differ less than a factor of 0.2 from the width of the broad Mg II lines since they are both forming in the same region and the broadening is dominated by global and not atomic parameters. The relative intensity of the individual intensities is set by the calculated oscillator strengths by Kurucz (1988) and Fuhr et al. (1988). 


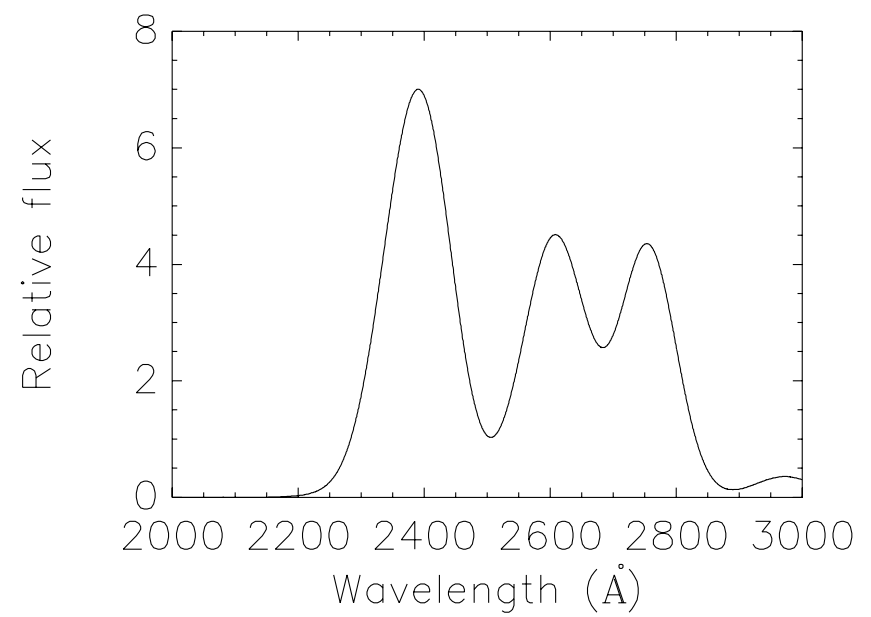

Fig. 3. Theoretical summed profile of the collisionally excited Fe II lines. In this plot the FWHM of the Fe II lines $\sqrt{M_{\mathrm{Mg}} / M_{\mathrm{Fe}}}$ times the width of the broad Mg II lines as observed in the STIS spectra. The relative intensity is such that the peak intensity of the Fe II lines with highest $A$ value, the $\lambda 2382.77\left({ }^{5} \mathrm{D}\right) 4 \mathrm{~s}^{6} \mathrm{D}_{9 / 2}-\left({ }^{5} \mathrm{D}\right) 4 \mathrm{p}{ }^{6} \mathrm{~F}_{11 / 2}$, is unity.

\subsection{Fe II fluorescence}

The H Ly $\alpha$ and the C IV resonance doublet at $1550 \AA$ are the strongest emission lines in the entire UV spectrum of NGC 4151. Both lines are very broad and originate from the same regions as the broad emission from Fe II (Peterson et al. 2004). This means that there are many $\mathrm{Fe}^{+}$ions subjected to substantial C IV and H Ly $\alpha$ emission in the central parts of this galaxy. This radiation in Fe II plasma can result in a selective population of specific Fe II levels (Johansson 1983) that decay, forming several lines in the 2000 to $3000 \AA$ region. To estimate the possibility of pumping, to a certain degree the atomic properties of the pumping channel as well as the nature of the pumping line have to be taken into account. I used the same equation as Eriksson et al. (2005) to estimate the relative pumping rates through the channels pumped by the same line,

$R \propto I_{\mathrm{p}} \cdot A_{\mathrm{c}} \cdot \mathrm{e}^{-\frac{\left(\lambda_{\mathrm{p}}-\lambda_{\mathrm{c}}\right)^{2}}{w_{\mathrm{p}}^{2}+w_{\mathrm{c}}^{2}}} \cdot \mathrm{e}^{-\frac{E_{l}}{k T}}$,

$R$ is the pumping rate, $I_{\mathrm{p}}$ the peak intensity of the pumping line, $A_{\mathrm{c}}$ the Einstein coefficient of the pumped channel and $E_{1}$ the lower energy of the lower level in the pumped channel. I calculated the relative population rate of the channels within the following three criteria. Their wavelength is within $10 \AA$ of any of the H Ly $\alpha$ and C IV $\lambda 1550$ doublet. The corresponding lower levels are less excited than $1 \mathrm{eV}$. The Einstein coefficient for the channels is higher than $10^{4} \mathrm{~s}^{-1}$. The widths used for the pumping lines are from Gaussian fits of their profiles from the STIS spectra. Since the C IV and Fe II emission both are believed to originate from the BLR, the width of the pumping channel is set to $\sqrt{M_{\mathrm{C}} / M_{\mathrm{Fe}}}$ times the observed width of the $\mathrm{C}$ IV line. H Ly $\alpha$ pumping of Fe II to $\left({ }^{5} \mathrm{D}\right) 5 \mathrm{p}$ levels have been observed in the symbiotic star RR Tel (Hartman \& Johansson 2000). Since Eq. (3) gives high relative pumping rates to the $\left({ }^{5} \mathrm{D}\right)$ levels, those levels can also be populated in the BLR of NGC 4151. However, a major factor of the resulting fluorescence lines from the population of the $5 \mathrm{p}$ levels is at IR wavelength, which is why I only considered the possible pumping to $4 \mathrm{p}$ levels. For all reasonable temperatures $(3000$ to $10000 \mathrm{~K})$ the relative pumping rate through the channels $3 \mathrm{~d}^{7}{ }^{4} \mathrm{~F}_{9 / 2}-\left({ }^{3} \mathrm{G}\right) 4 \mathrm{p}{ }^{4} \mathrm{H}_{11 / 2}$ and ( $\left.{ }^{5} \mathrm{D}\right) 4 \mathrm{~s}{ }^{4} \mathrm{D}_{7 / 2}-\left({ }^{3} \mathrm{~F}\right) 4 \mathrm{p}{ }^{4} \mathrm{G}_{9 / 2}$ at $\lambda 1218.21$ is more than a magnitude higher than for any of the other possible pumping channels
Table 3. Relative intensity of the Fe II fluorescence lines.

\begin{tabular}{lccc}
\hline \hline Pumped level & Wavelengt $(\AA)$ & Lower level & Intensity \\
\hline$\left({ }^{3} \mathrm{G}\right) 4 \mathrm{p}{ }^{4} \mathrm{H}_{11 / 2}$ & 2772.00 & $\left({ }^{3} \mathrm{G}\right) 4 \mathrm{~s}^{2} \mathrm{G}_{9 / 2}$ & $0.60 \mathrm{I}_{2458}$ \\
& 2493.10 & $\left({ }^{3} \mathrm{H}\right) 4 \mathrm{~s}^{2} \mathrm{H}_{9 / 2}$ & $0.81 \mathrm{I}_{2485}$ \\
& 2481.80 & $\left({ }^{3} \mathrm{H}\right) 4 \mathrm{~s}^{2} \mathrm{H}_{11 / 2}$ & $0.65 \mathrm{I}_{2485}$ \\
& 2459.53 & $\left({ }^{3} \mathrm{G}\right) 4 \mathrm{~s}^{4} \mathrm{G}_{9 / 2}$ & $\mathrm{I}_{2485}$ \\
& 2436.96 & $\left({ }^{3} \mathrm{G}\right) 4 \mathrm{~s}^{4} \mathrm{G}_{11 / 2}$ & $0.55 \mathrm{I}_{2485}$ \\
& 2220.59 & $\left({ }^{3} \mathrm{H}\right) 4 \mathrm{~s}^{4} \mathrm{H}_{11 / 2}$ & $0.43 \mathrm{I}_{2485}$ \\
\hline$\left({ }^{3} \mathrm{~F}\right) 4 \mathrm{p}{ }^{4} \mathrm{G}_{9 / 2}$ & 2509.10 & $\left({ }^{3} \mathrm{G}\right) 4 \mathrm{~s}^{2} \mathrm{G}_{9 / 2}$ & $\mathrm{I}_{2509}$ \\
& 2507.19 & $\left({ }^{3} \mathrm{G}\right) 4 \mathrm{~s}^{2} \mathrm{G}_{7 / 2}$ & $0.11 \mathrm{I}_{2509}$ \\
\hline
\end{tabular}

Notes. $\mathrm{I}_{2509}$ is the intensity of the $\lambda 2509.10$ line and $\mathrm{I}_{2459}$ is the intensity of the $\lambda 2459.53$ line.

in NGC 4151. Therefore pumping in those to channels is incorporated in my model. Pumping to the $\mathrm{y}^{4} \mathrm{H}_{11 / 2}\left(\left({ }^{3} \mathrm{G}\right) 4 \mathrm{p}{ }^{4} \mathrm{H}_{11 / 2}\right)$ by the $\mathrm{C}$ IV resonance doublet was the first observed PAR process involving Fe II observed in symbiotic stars (Johansson 1983). Now pumping to the Fe II $\mathrm{y}^{4} \mathrm{H}_{11 / 2}$ and $\left({ }^{3} \mathrm{~F}\right) 4 \mathrm{p}{ }^{4} \mathrm{G}_{9 / 2}$ levels has been observed in several objects (Eriksson et al. 2004). It is therefore not surprising that Eq. (3) indicates that those levels are the most probable PAR pumped levels also in the BLR of NGC 4151. In the model spectra the width of the Fe II fluorescence lines is the same as for the collisionally excited lines. Oscillator strengths associated with high parent terms in complex ions are difficult to calculate with precision. The calculated strengths for the $4 s-4 p$ transitions of high parent terms differ by magnitudes between different calculations. I therefore instead used the spectra of symbiotic stars to estimate the relative intensity of the fluorescence lines from $y^{4} \mathrm{H}_{11 / 2}$. Table 3 lists the relative intensities for the fluorescence lines as modeled in NGC 4151. The relative intensities are based on the average relative intensity as measured in symbiotic stars. For the fluorescence lines from the $\left({ }^{3} \mathrm{~F}\right) 4 \mathrm{p}{ }^{4} \mathrm{G}_{9 / 2}$ level the calculated values are a better choice in spite of the poor precision. This is because one of the resulting lines, 22509.10 , has branching fraction higher by one magnitude than any other line in the 2000-3000 $\AA$ region. The other lines have therefore rarely been observable in symbiotic stars, and when they are observed, their intensity is difficult to measure with good precision. For the lines from the $\left({ }^{3} \mathrm{~F}\right) 4 \mathrm{p}^{4} \mathrm{G}_{9 / 2}$ level calculations from Kurucz (1988) were used.

\section{Results}

\subsection{Excluding fluorescence}

To see if the PAR mechanism should be incorporated in the model spectra, the best fit of the model spectra excluding fluorescence were analyzed. Figure 4 presents the best possible fit to the NGC 4151 STIS spectra without incorporating PAR. It is clear purely from topology that there is emission in the middle of the 2000-3000 $\AA$ region that is not explained by my model spectra without fluorescence. For a more quantitative comparison with the best fits including fluorescence the mean fractional difference (Eq. (4)) between observed data and best fits was calculated, see Table 4. In the next section I show how this excess in emission is fitted with the Fe II fluorescence discussed in Sect. 3.3,

$m d=\frac{\left\langle\left|I_{\mathrm{obs}}-I_{\text {model }}\right|\right\rangle}{\left\langle\left|I_{\mathrm{obs}}\right|\right\rangle}$ 


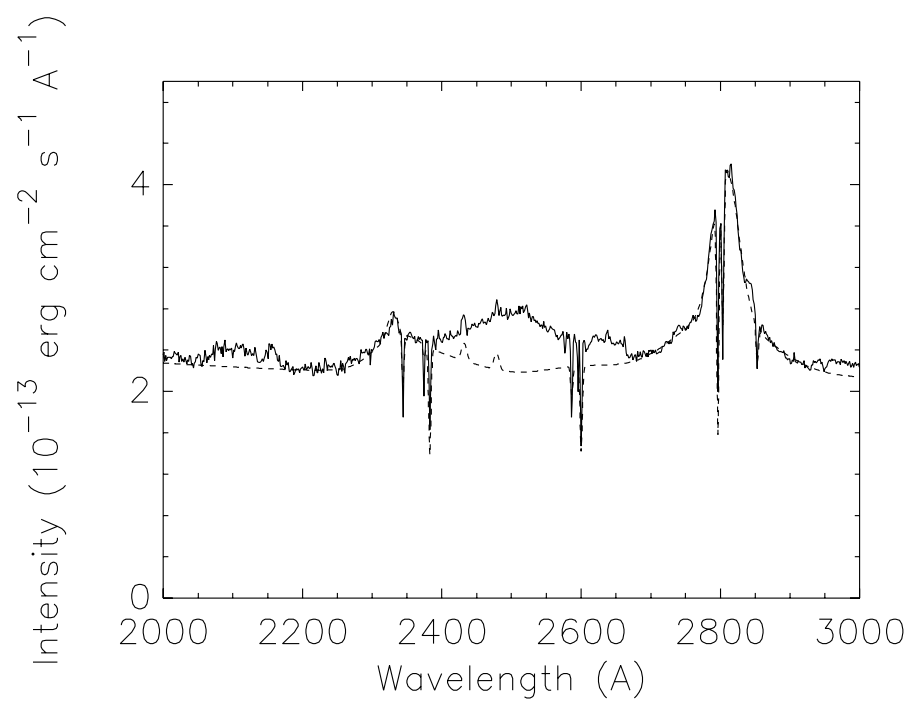

Fig. 4. Best possible fit excluding Fe II fluorescence. The solid line is the STIS spectrum o42305070 and the dotted line is the theoretical spectrum.

Table 4. Mean fractional difference observed to model spectra.

\begin{tabular}{lcc}
\hline \hline STIS Spectra & $m d$ without fluorescence & $m d$ with fluorescence \\
\hline o42303070 & 0.078 & 0.030 \\
042305070 & 0.074 & 0.035 \\
059701040 & 0.075 & 0.034 \\
\hline
\end{tabular}

\subsection{Including fluorescence}

There is a clear excess of flux between 2400 and $2600 \AA$ in the NGC 4151 spectrum, see Fig. 4. This bump is nicely fitted by incorporating fluorescence, see Fig. 5. Furthermore, from comparisons of the mean fractional difference between fit and observations including or excluding Fe II fluorescence (Table 4), the PAR process are shown to be active in central parts of NGC 4151. The remaining mean fractional difference after including fluorescence is mostly due to two unidentified features, one ranging from 2060 to $2160 \AA$ and one ranging from 2600 to $2660 \AA$.

The forbidden lines, Table 2, have a FWHM of about $200 \mathrm{~km} \mathrm{~s}^{-1}$ (Nagao et al. 2003), which is why they are modeled as STIS instrumental profiles in the theoretical spectra. The flux of the individual forbidden lines is the same in all three STIS spectra used in this study. This is expected since the forbidden emission does not originate from the surrounding clouds (Kaiser et al. 2000) and should have a nature independent of position angle. Since the continuum is weaker at lower position angle (Nelson et al. 2000), the forbidden lines have a higher $\mathrm{S} / \mathrm{N}$ ratio in the two STIS spectra with lower position angle. The width of the C II intercombination line is slightly broader in the best fits than that of the NLR components of the Mg II lines. I therefore suggest that the $\mathrm{C}$ II intercombination lines are formed in the same region as the Mg II lines. This would imply a wide range of electron densities for this regions since the lifetimes for the C II $2 s 2 p{ }^{4} \mathrm{P}$ levels are several magnitudes higher than those of the Mg II $3 p$ levels. It should be remembered, though, that the observed $\lambda 2326$ line is a blend between the C II intercombination doublet and a forbidden [O III] line. It can still be concluded that the C II intercombination lines are far wider than those of the forbidden lines. The FWHM

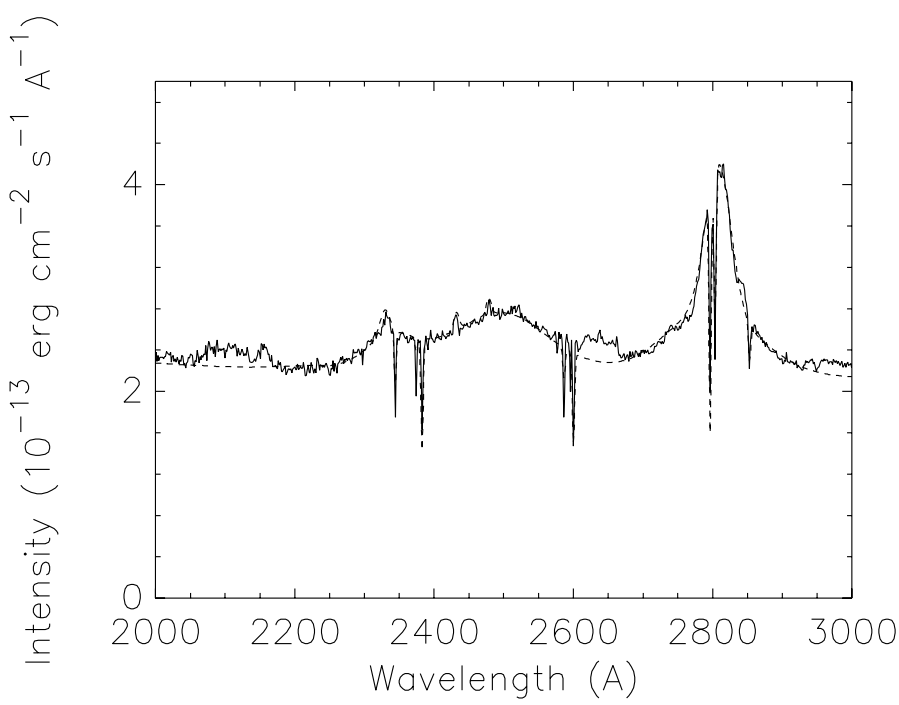

Fig. 5. Best possible fit including Fe II fluorescence. The solid line is the STIS spectrum 042305070 and the dotted line is the theoretical spectrum.

Table 5. Integrated intensities of the emission lines in the 2000 to 3000 Å region.

\begin{tabular}{l|ccc}
\hline \hline & \multicolumn{3}{|c}{ Flux $\left(10^{-13} \mathrm{erg} \mathrm{cm}^{-2} \mathrm{~s}^{-1}\right)$} \\
& 042303070 & 042305070 & 059701040 \\
\hline$\left[\mathrm{O}\right.$ III $2 \mathrm{p}^{3}{ }^{4} \mathrm{~S}-2 \mathrm{p}^{3}{ }^{2} \mathrm{P}$ & 2.01 & 0.26 & 0.26 \\
{$\left[\mathrm{O}\right.$ III $2 \mathrm{p}^{2}{ }^{3} \mathrm{P}_{1}-2 \mathrm{p}^{2}{ }^{1} \mathrm{~S}_{0}$} & 5.93 & 5.07 & 1.00 \\
{$\left[\mathrm{Ne}\right.$ IV] $2 \mathrm{p}^{3}{ }^{4} \mathrm{~S}-2 \mathrm{p}^{3}{ }^{2} \mathrm{D}$} & 4.02 & 2.30 & 0.56 \\
\hline $\mathrm{C}$ II $2 \mathrm{~s}^{2} 2 \mathrm{p}^{2} \mathrm{P}-2 \mathrm{~s} 2 \mathrm{p}^{2}{ }^{4} \mathrm{P}$ & 27.5 & 7.83 & 2.86 \\
$\mathrm{Mg}$ II Inremediate component & 91.5 & 40.2 & 11.6 \\
\hline $\mathrm{Mg}$ II Broad component & 128 & 42.8 & 11.0 \\
Fe II collsionally excited & 87.2 & 18.8 & $<5$ \\
Fe II PAR excited & 138 & 41.4 & 8.76 \\
\hline Continuum 2000-3000 $\AA$ & 2120 & 230 & 218 \\
\hline
\end{tabular}

of the broad lines in our best fits was $15000 \mathrm{~km} \mathrm{~s}^{-1}$ (Fe II) and $17500 \mathrm{~km} \mathrm{~s}^{-1}$ (Mg II broad components). The similar widths of these lines were expected since the ionization and excitation energies needed to produce those lines are similar. The total integrated emission from BLR, Fe II collision excited lines, Fe II fluorescence lines and Mg II broad components are presented in Table 5. The emission from ILR and NLR, different forbidden lines, the C II intercombination lines, and the $\mathrm{Mg}$ II narrower components are also presented in Table 5.

\section{Conclusions}

The PAR is present in the BLR of NGC 4151 because the C IV $\lambda 1550$ doublet and H Ly $\alpha$ are pumping Fe II to the highly excited levels $\left({ }^{3} \mathrm{G}\right) 4 \mathrm{p}{ }^{4} \mathrm{H}_{11 / 2}$ and $\left({ }^{3} \mathrm{~F}\right) 4 \mathrm{p}{ }^{4} \mathrm{G}_{9 / 2}$. The total flux of the Fe II fluorescence lines is the strongest emission contribution in the 2000-3000 $\AA$ region after the Mg II $\lambda 2800$ doublet. It contributes 20 to 30 percent of the total emission line flux and about 5 percent of the total flux including continuum in the investigated wavelength region. The fluorescence lines are energy absorbed from the pumping lines, which still escape the system and can thus have a cooling effect on the BLR. In addition to the fluorescence lines, Fe II is also emitting lines from the 25 collisionally excited $\left({ }^{5} \mathrm{D}\right) 4 \mathrm{p}$ levels. The PAR and collisionally excited 
lines from Fe II make this the element that radiates the highest emission line flux in the investigated wavelength region. The ILR emission components first detected by Kraemer et al. (2006) account for approximately half of the emission of the $\mathrm{Mg}$ II resonance doublet. Interestingly, the intercombination multiplet of C II at $2430 \AA$ can only be modeled by assuming it to be dominated by ILR radiation. The lifetime of the C II levels involved ranges from the shorter ones for the allowed NLR transitions to the longer ones for the forbidden NLR transitions. I suggest that the ILR is just an extension of the BLR, because moving outward from the disk within the disk wind of NGC 4151, the electron density continuously decreases. The longer the lifetime of the upper levels, the farther away from the disk the lines are formed.

\section{References}

Baldwin, J. A., Ferland, G. J., Korista, K. T., Hamann, F., \& LaCluyze, A. 2004, ApJ, 615, 610

Bentz, M. C., Denney, K. D., Cackett, E. M., et al. 2006, ApJ, 651, 775

Chiang, J., \& Murray, N. 1996, ApJ, 466, 704

Crenshaw, D. M., \& Kraemer, S. B. 2001, ApJ, 562, 29

Crenshaw, D. M., \& Kraemer, S. B. 2005, ApJ, 625, 680
Crenshaw, D. M., \& Kraemer, S. B. 2007, ApJ, 659, 250

Crenshaw, D. M., Kraemer, S. B., Hutchings, J. B., et al. 2000, ApJ, 545, 27 Eriksson, M., Veenhuizen, H., Wahlgren, G. M., \& Johansson, S. 2004, IAU Colloq., 191, 132

Eriksson, M., Johansson, S., Wahlgren, G. M., et al. 2005, A\&A, 434, 397

Eriksson, M., Johansson, S., \& Wahlgren, G. M. 2006, A\&A, 451, 157

Fuhr, J. R., Martin, G. A., \& Wiese, W. L. 1988, J. Phys. Chem. Ref. Data, 17, Suppl. 4

Gregory, A. S., Randi, R. L., \& Salviander, S. 2010, ApJ, 721, 1835

Halenka, J., \& Madej, J. 2002, Acta Astron., 52, 195

Hartman, H., \& Johansson, S. 2000, A\&A, 359, 627

Hutchins, J. B. 1998, HST proposal, 7569

Johansson, S. 1983, MNRAS, 205, 71

Kaiser, M. E., Bradley, L. D., Hutchings, J. B., et al. 2000, ApJ, 528, 260

Kraemer, S. B., Geroge, I. M., Crenshaw, D. M., et al. 2005, ApJ, 633, 693

Kraemer, S. B., Crenshaw, D. M., Gabel, J. R., et al. 2006, ApJS, 167, 161

Kurucz, R. L. 1988, IAU Colloq., 94, 41

Leech, K. J., Penston, M. V., Snijders, M. A. J., \& Gull, T. R. 1987, MNRAS, 225,837

Mundell, C. G. 2000, HST proposal, 8136

Nagao, T., Murayama, T., Shioya, Y., \& Taniguchi, Y. 2003, AJ, 126, 1167

Nelson, C. H., Weistrop, D., Hutchings, J. B., et al. 2000, ApJ, 531, 257

Peterson, B. M., Ferrarese, L., Gilbert, K. M., et al. 2004, ApJ, 613, 682

Smith, R. J., Lucey, J. R., Hudson, M. J., \& Bridges, T. J. 2009, MNRAS, 398, 119

Savage, B. D., \& Mathis, J. S. 1979, ARA\&A, 17, 73

Woodgate, B. E., Kimble, R. A., Bowers, C. W., et al. 1998, PASP, 110, 1183 